\title{
Caractéristiques biophysiques du bois de Pterocarpus erinaceus (Poir.) en zones guinéenne et soudanienne au Togo
}

Kossi Novinyo SEgLA ${ }^{1}$ Adzo Dzifa KoкUTSE ${ }^{1}$ Kossi AdjONOU ${ }^{1}$ Patrick LANGBOUR ${ }^{2}$ Gilles ChAIX ${ }^{3}$

Daniel GUIBAL 2 Kouami KoKou ${ }^{1}$

1 Université de Lomé Faculté des sciences Laboratoire de botanique et écologie végétale BP 1515, Lomé Togo

${ }^{2}$ Cirad

Département PERSYST

URP BioWooEB

34398 Montpellier Cedex 5 France

${ }^{3}$ Cirad

Département BIOS UMR AGAP

34398 Montpellier Cedex 5 France

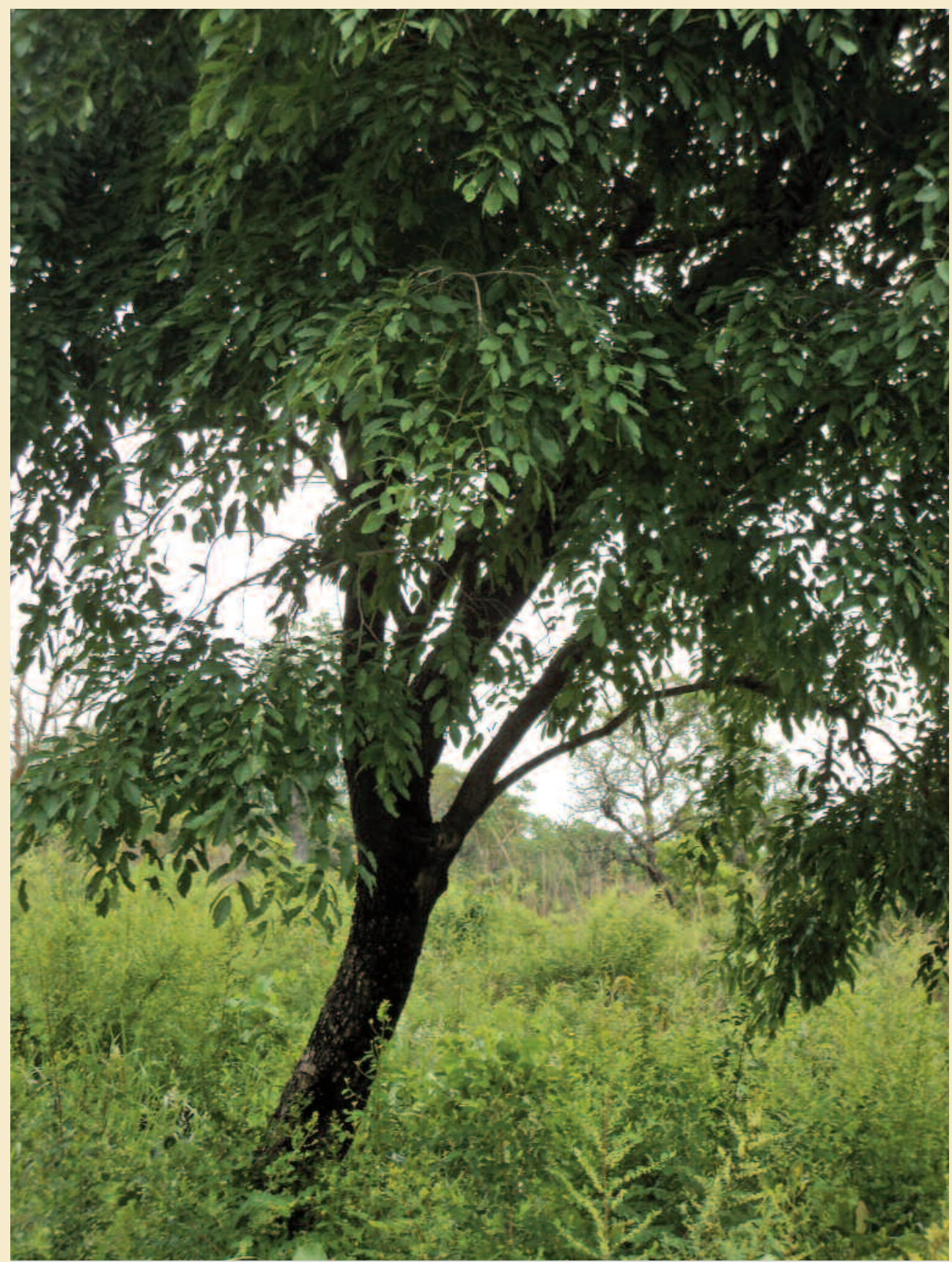

Photo 1.

Pied de Pterocarpus erinaceus dans son milieu naturel au Togo. Photo K. N. Segla. 


\section{RÉSUMÉ}

\section{CARACTÉRISTIQUES BIOPHYSIQUES DU BOIS DE PTEROCARPUS ERINACEUS (POIR.) EN ZONES GUINÉENNE ET SOUDANIENNE AU TOGO}

Pterocarpus erinaceus (Poir.) est l'une des espèces des domaines climatiques soudanien et guinéen du Togo. Son importance socio-économique tient à ses nombreuses utilisations. Toutefois, la sylviculture et les propriétés technologiques du bois sont mal connues. C'est dans ce contexte qu'un travail a été mené pour déterminer les propriétés physiques et mécaniques (densité, retraits, PSF, MOE, MOR, compression, dureté) de son bois en relation avec les conditions du milieu. Les résultats observés sur les bois de 19 arbres, âgés de 18 à 60 ans environ, prélevés dans deux zones écologiques (Parc de la Kéran, zone soudanienne; Abdoulaye, zone guinéenne), montrent des différences de densité, d'infra-densité, de contrainte de rupture en compression et d'anisotropie des retraits. Ces propriétés sont plus élevées à Kéran qu'à Abdoulaye. Pour les retraits, le PSF, le MOE, la contrainte de rupture en flexion et la dureté, il n'y a pas de différence significative entre les deux sites. Cette étude met en évidence des corrélations attendues entre la densité et la dureté. Elle montre aussi que les propriétés physiques et mécaniques du bois de vène sont très peu influencées par l'âge cambial.

Mots-clés : Pterocarpus erinaceus, propriétés physiques et mécaniques, milieux, Togo.

\section{ABSTRACT}

\section{BIOPHYSICAL CHARACTERISTICS OF PTEROCARPUS ERINACEUS (POIR.) TIMBER IN TOGO'S GUINEAN AND SUDANIAN ZONES}

Pterocarpus erinaceus (Poir.) is a tree species found in the Soudanian and Guinean climatic zones in Togo. It is of high socio-cultural importance because of its many uses, but little is known about the technological properties of its wood. This is why a study was conducted to determine the physical and mechanical properties (density, shrinkage, FSP, MOE, MOR, compression, hardness) of its wood in relation to environmental conditions. The results for the wood of 19 trees of about 18 to 60 years of age, growing in two ecological zones (Keran Park in the Sudanian zone and Abdoulaye in the Guinean zone), show that density, infradensity, compressive yield stress and anisotropic shrinkage are all higher in the Keran trees. Regarding shrinkage, FSP, MOE, tensile strength and hardness, no significant differences were found between the two sites. The expected correlations were found between density and hardness. The study also shows that the physical and mechanical properties of Pterocarpus erinaceus wood are virtually unaffected by cambium age.

Keywords: Pterocarpus erinaceus, physical and mechanical properties, environments, Togo.

\section{RESUMEN}

\section{CARACTERÍSTICAS BIOFÍSICAS DE LA MADERA DE PTEROCARPUS ERINACEUS (POIR.) EN LA ZONA GUINEANA Y SUDANESA DE TOGO}

Pterocarpus erinaceus (Poir.) es una de las especies de los dominios climáticos sudanés y guineano de Togo. Su importancia socioeconómica se deriva de sus muchos usos. Sin embargo, la silvicultura y las propiedades tecnológicas de la madera son mal conocidas. En este contexto, se llevó a cabo un estudio para determinar las propiedades físicas y mecánicas (densidad, contracciones, PSF, MOE, MOR, compresión, dureza) de su madera en relación con las condiciones ambientales. Los resultados observados en la madera de 19 árboles, de unos 18 a 60 años de edad, sacados de dos áreas ecológicas (Parque del Kéran, zona sudanesa y Abdoulaye, zona guineana), muestran diferencias de densidad, densidad básica, esfuerzo de ruptura en compresión y anisotropía de las contracciones. Estas propiedades son más altas en Kéran que en Abdoulaye. Respecto de las contracciones, PSF, MOE, esfuerzo de ruptura en flexión y dureza, no hay diferencias significativas entre ambos sitios. Este estudio evidencia las esperadas correlaciones entre densidad y dureza. Asimismo, se demuestra que las propiedades físicas y mecánicas de la madera de Pterocarpus erinaceus se ven muy poco influidas por la edad cambial.

Palabras clave: Pterocarpus erinaceus, propiedades físicas y mecánicas, medio ambiente, Togo. 


\section{Introduction}

La réduction des superficies forestières au Togo se traduit par une variation annuelle du couvert forestier estimée à près de 5,1 \%, (FAO, 2011). La situation est aujourd'hui encore plus préoccupante car l'exploitation forestière des formations naturelles se pratique en ignorant les principes d'aménagement forestier durable. Le prélèvement sélectif de certaines espèces de bois d'œuvre de qualité menace dangereusement les ressources phytogénétiques du pays. Parmi les espèces ainsi menacées se trouve le vène, Pterocarpus erinaceus (Poir.) (Fabaceae), espèce inféodée aux zones tropicales sahéliennes arides et soudaniennes semi-arides. Elle est exploitée fortement au Togo comme bois d'œuvre et bois de service (Adjonou et al., 2010). En 2008, le Togo a exporté près de $3500 \mathrm{~m}^{3}$ de madriers à base de $P$. erinaceus (MERF/OIBT, 2010). La quasi-totalité de ce bois est exportée vers la Chine sous forme de grumes. Cette espèce est également très utilisée pour la production de bois énergie de première catégorie au Togo (Kokou et al., 2009).

$P$. erinaceus revêt une grande importance par son bois de qualité à usage multiple et par les usages traditionnels (pharmacopée, source de tanin) qu'il permet. Selon des études effectuées par le Cirad sur le bois de quelques arbres, $P$. erinaceus possède un bois dense avec un retrait linéaire très faible associé à un point de saturation très bas. C'est une espèce mécaniquement très résistante et très durable. Hormis les travaux réalisés sur du bois de vène du Mali (Traoré, 2009 ; Traoré et al., 2010), il existe peu de données fiables sur la qualité du bois en relation avec ses caractéristiques structurelles (rythme de croissance, anatomie, extractibles) et avec les conditions environnementales de croissance.

Au Togo, on observe le plus souvent un usage inapproprié, souvent sous-valorisé, du bois de cette espèce. Celleci est aujourd'hui menacée par une forte pression anthropique et fait partie des espèces justifiant des actions prioritaires, notamment l'orientation vers la sylviculture et l'intégration dans les programmes de reboisement.

C'est dans cette perspective que la présente étude a été réalisée. Il s'agissait, au Togo, d'analyser, d'une part, l'influence de la provenance de $P$. erinaceus, en interaction avec les conditions environnementales, sur le comportement physique et mécanique du bois et, d'autre part, l'influence de la densité et de l'âge sur ces propriétés.

\section{Matériel et méthode}

\section{Zones d'étude}

\section{Réserve de faune d'Abdoulaye (zone guinéenne)}

La réserve de faune d'Abdoulaye est située entre les longitudes $1^{\circ} 15^{\prime}$ et $1^{\circ} 27^{\prime}$ 'Est et entre les latitudes $8^{\circ} 33^{\prime}$ et $8^{\circ} 47^{\prime}$ Nord dans la zone écologique III du Togo (figure 1). Cette zone bénéficie d'un climat relativement nuancé allant du subéquatorial de moyenne altitude au climat tropical semi-humide qui se caractérise par deux saisons distinctes : une saison pluvieuse et une saison sèche. La pluviométrie varie entre 1200 et $1300 \mathrm{~mm}$ par an. Selon le service de météorologie de la région centrale, la température moyenne mensuelle est de $26^{\circ} \mathrm{C}$.

\section{Parc national Oti-Kéran (zone soudanienne)}

Le Parc national Oti-Kéran est situé dans la région septentrionale du Togo, entre $9^{\circ} 55^{\prime}$ et $10^{\circ} 20^{\prime}$ de latitude Nord et $0^{\circ} 25^{\prime}$ et $1^{\circ} 00^{\prime}$ de longitude Est, dans la zone écologique I (figure 1). Le climat de type soudanien est caractérisé par une saison pluvieuse et une saison sèche, marquée par l'harmattan. Les précipitations totales annuelles sont de l'ordre de $1200 \mathrm{~mm}$ à Kanté et de $1050 \mathrm{~mm}$ à Mango. La moyenne thermique mensuelle de la région se situe autour de $31,5^{\circ} \mathrm{C}$.

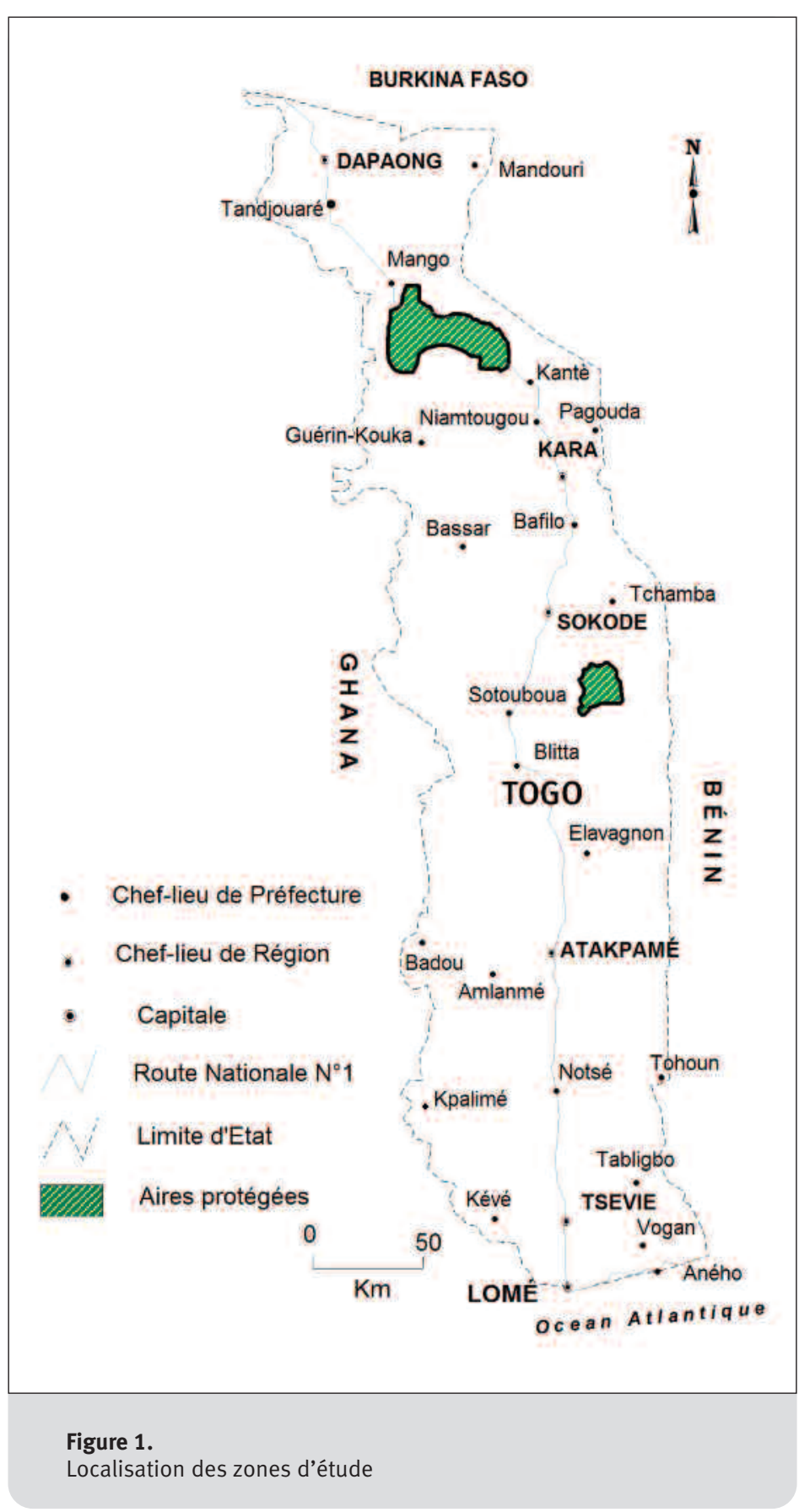




\section{Méthodologie}

\section{Échantillonnage et réalisation des éprouvettes}

Les prélèvements de bois ont été effectués dans la forêt d'Abdoulaye et dans la réserve de la Kéran. Ces prélèvements ont été réalisés sans tenir compte du diamètre ni de la hauteur des arbres, mais en ne retenant que les arbres bien conformés. L'environnement de chaque arbre a été caractérisé par des descripteurs écologiques tels que le climat, la pluviométrie, le type de sol et le type de végétation. Dix arbres ont été prélevés sur le site d'Abdoulaye, et neuf sur le site de la Kéran. Pour chaque arbre ont été mesurées les données dendrométriques telles que la hauteur du fût, la hauteur totale et la circonférence à la base et à 1,30 m. À une hauteur de $1,30 \mathrm{~m}$ pour chaque arbre, un billon d'environ $1 \mathrm{~m}$ a été prélevé et un plateau diamétral, allant de la moelle à l'écorce, a été découpé dans le billon.

Au total, 19 plateaux de $60 \mathrm{~cm}$ de longueur et de $5 \mathrm{~cm}$ d'épaisseur ont été recueillis. Leur largeur dépend du diamètre des troncs. Les plateaux ont été acheminés au Cirad, à Montpellier. Afin de considérer la variabilité des propriétés du bois de la moelle vers l'écorce, des éprouvettes ont été prélevées dans le duramen, tangentiellement aux cernes d'accroissement, puis débitées en ébauches et placées jusqu'à stabilisation de leur masse dans une chambre climatisée $\left(\mathrm{T}=20^{\circ} \mathrm{C} \pm 2{ }^{\circ} \mathrm{C}\right.$ et $\left.\mathrm{Hr}=65 \% \pm 5 \%\right)$. À partir de ces ébauches, une série d'éprouvettes de dimensions $2 \times 2 \times 1 \mathrm{~cm}, 2 \times 2 \times 6 \mathrm{~cm}$ et $2 \times 2 \times 36 \mathrm{~cm}$ ont été confectionnées suivant les orientations radiale ( $R$ ), tangentielle (T) et longitudinale $(L)$ respectivement selon les normes françaises en vigueur (NF B51-002) (figure 2). Les propriétés physiques et mécaniques de référence, à savoir la masse volumique, l'infra-densité, les retraits tangentiel et radial et le module d'élasticité longitudinale, la compression axiale, la résistance à la flexion statique et la dureté, ont été mesurées sur ces éprouvettes.
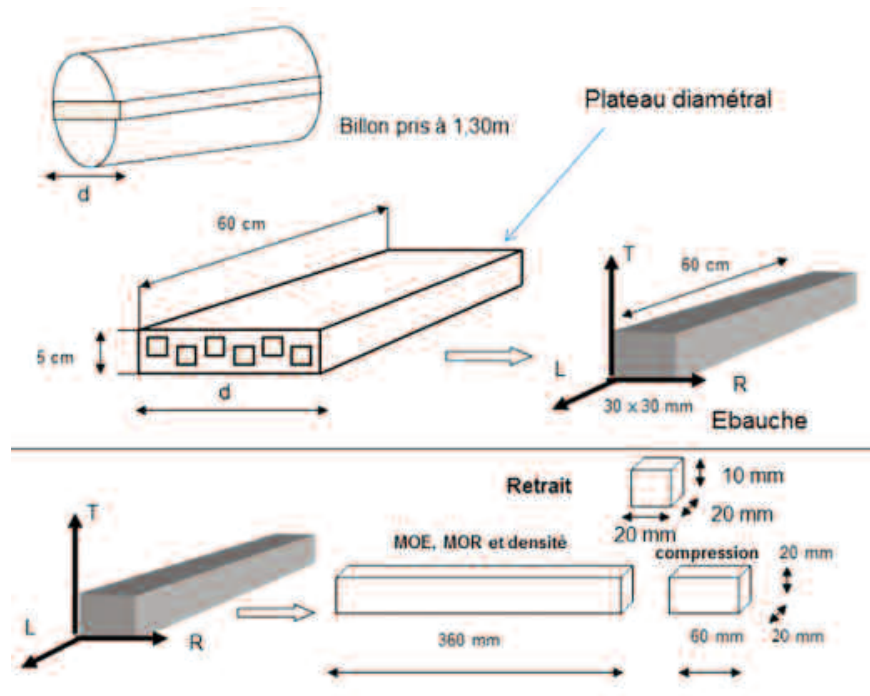

Figure 2.

Détails sur les découpes des plateaux et éprouvettes à partir des billons.

\section{Détermination de l'âge des échantillons}

L'âge a été déterminé par comptage des cernes d'accroissement annuel sur une tranche de bois découpée transversalement dans chaque plateau et préalablement poncée au papier de verre de grain 400. L'échantillon a été disposé sous une loupe binoculaire (Leitz, grossissement 8 à 35) pour une lecture des éléments du plan ligneux dans le plan transversal. Les cellules de parenchyme axial permettaient de délimiter des cernes d'accroissement par des alternances de bandes plus épaisses, plus courtes et sinueuses en début d'accroissement, plus fines et plus rectilignes en fin d'accroissement. Sur quelques arbres, l'étroitesse des cernes rendait le décompte difficile; un double comptage a permis d'évaluer l'âge à plus ou moins un an près.

\section{Mesure de la densité à $12 \%$ d'humidité du bois et de l'infra-densité (densité basale)}

La densité est le rapport entre la masse et le volume de l'éprouvette et est exprimée en grammes par centimètre cube $\left(\mathrm{g} / \mathrm{cm}^{3}\right)$ pour une humidité donnée. La masse de chaque éprouvette a été déterminée par une balance de résolution $0,01 \mathrm{~g}$, et les dimensions (longueur, largeur et épaisseur) permettant de calculer son volume ont été mesurées à l'aide d'un pied à coulisse électronique d'une précision de 0,01 mm. L'infra-densité, ou densité basale (en $\mathrm{kg} / \mathrm{m}^{3}$ ), est le rapport entre la masse anhydre de chaque éprouvette (obtenue après séchage à $103^{\circ} \mathrm{C}$ ) et son volume saturé mesuré selon le principe d’Archimède.

\section{Mesure du module d'élasticité longitudinal}

Le module d'élasticité longitudinal (MOE) a été déterminé selon la technique de la vibration libre sur poutres flottantes par usage du dispositif appelé «Bing » (Beam Identification by Non destructive Grading). Ce dispositif développé au Cirad permet de déterminer les caractéristiques physico-mécaniques des échantillons de bois par simple excitation vibratoire (Bordonne, 1989). La méthode repose sur la recherche puis l'interprétation de spectre des fréquences propres issues des vibrations naturelles en appliquant la théorie développée par Bernouilli et reprise par Timoshenko et Goodier (1951). Le module spécifique a été calculé en réalisant le rapport entre le MOE et la densité.

\section{Dureté Monnin}

Les essais de dureté Monnin ont été réalisés sur les éprouvettes préparées pour mesurer le module d'élasticité, selon la norme française NF B51-013 (AFNOR, 1988). Le principe est de déterminer la résistance à la pénétration sur la face radiale du bois d'un cylindre métallique de rayon donné, appliqué suivant une génératrice sous un effort continu jusqu'à la charge maximale de $1960 \mathrm{~N}$. Une feuille de papier carbone interposée entre le cylindre et l'éprouvette permet d'obtenir une empreinte visible. Cette largeur d'empreinte a été mesurée au moyen d'un comparateur optique de façon à déterminer la valeur $\mathrm{N}$ de la dureté Monnin. 


\section{Essai de rupture en flexion statique (MOR)}

Sur les éprouvettes valorisées par la méthode « Bing », nous avons effectué des essais de flexion statique quatre points dont le principe est décrit par la norme NF B51-008. Cette méthode permet la détermination de la contrainte de rupture par flexion sous une charge progressivement croissante, exercée perpendiculairement au fil du bois. Les essais ont été réalisés à l'aide d'une machine d'essais universelle MTS d'une précision de $1 \%$ sur la mesure de la charge.

\section{Essai de rupture en compression axiale}

Des éprouvettes de dimensions géométriques $2 \times 2 \times 6 \mathrm{~cm}$ suivant les directions R, T, L ont été soumises aux essais de compression axiale à l'aide d'une machine universelle avec une cellule de charge maximale égale à 10 tonnes. La procédure décrite dans la norme NF B51-008 permet d'obtenir les valeurs des déplacements et des charges jusqu'à la rupture de l'éprouvette. L'effort maximum obtenu donne accès à la contrainte à la rupture en compression axiale.

Retraits radial et tangentiel, retrait surfacique, coefficient de retrait surfacique et point de saturation des fibres (PSF)

À l'intérieur de chaque éprouvette ayant servi aux mesures de module d'élasticité et de densité, une éprouvette de dimensions $2 \times 2 \times 1 \mathrm{~cm}$ selon les directions $R, T, L$ a été prélevée. Ces éprouvettes ont été saturées par trempage dans de l'eau distillée pendant environ 48 h en subissant deux cycles de dépression-pression pour parfaire la saturation. Les éprouvettes ainsi saturées ont été pesées à l'aide d'une balance électronique pour obtenir la masse saturée. Les dimensions suivant les directions $\mathrm{R}$ et $\mathrm{T}$ à l'état saturé ont également été relevées. Le volume saturé a été mesuré sur une balance selon le principe d'Archimède. Les éprouvettes de bois ont ensuite été placées dans une enceinte climatique réglée en température et humidité relative de l'air, respectivement sur $30{ }^{\circ} \mathrm{C}-85 \% \mathrm{Hr}$, $20{ }^{\circ} \mathrm{C}-65 \% \mathrm{Hr}, 20^{\circ} \mathrm{C}-30 \% \mathrm{Hr}$, pour être ramenées successivement à une humidité du bois théorique de $18 \%, 12 \%, 6 \%$. Les dimensions et la masse ont été prises respectivement à $18 \%, 12 \%, 6 \%$ et $0 \%$. Pour passer de $6 \%$ d'humidité à l'état anhydre, les éprouvettes ont été maintenues à l'étuve pendant $48 \mathrm{~h}$ à une température de $103^{\circ} \mathrm{C}$. La teneur en eau (X \%), les retraits radial ( $\mathrm{Rr}$ ) et tangentiel (Rt) ont été calculés comme suit :

$\mathrm{X} \%=\frac{P S-P a}{P a} \times 100$

Avec :

Ps = poids des éprouvettes à l'état saturé ;

$\mathrm{Pa}=$ poids des éprouvettes à l'état anhydre ;

$X=$ teneur en eau de l'éprouvette en \%.

$\mathrm{Rr}=\frac{\text { Drs }- \text { Dra }}{\text { Drs }} \times 100$

Avec :

Drs = dimension radiale de l'éprouvette à l'état saturé ;

Dra $=$ dimension radiale de l'éprouvette à l'état anhydre ; $\mathrm{Rr}=$ retrait radial de l'éprouvette en \%.
$\mathrm{Rt}=\frac{D t s-D t a}{D t s} \times 100$

Avec :

Dts = dimension tangentielle de l'éprouvette saturée ;

Dta $=$ dimension tangentielle de l'éprouvette anhydre;

$\mathrm{Rt}=$ retrait tangentiel de l'éprouvette en \%.

À partir des retraits radial et tangentiel, le retrait surfacique a été calculé selon la formule $\mathrm{Rt}+\mathrm{Rr}+(\mathrm{Rt} \times \mathrm{Rr}) / 100)$; il est quasi identique au retrait volumique. Le coefficient de retrait surfacique a ensuite été déterminé en faisant le rapport entre le retrait surfacique et le PSF.

Le point de saturation des fibres (PSF) est une caractéristique importante du bois qui régit les changements de ses propriétés (Skaar, 1988). Il correspond à l'humidité en dessous de laquelle les propriétés physiques et mécaniques du bois évoluent en fonction de la teneur en humidité. Le PSF est déterminé en utilisant la fonction linéaire reliant les différences de surfaces aux humidités correspondantes (Guitard, 1987).

\section{Méthode d'analyse statistique}

L'analyse statistique des données mécaniques et physiques a été réalisée avec le logiciel R. L'objectif étant d'étudier la variabilité selon la provenance (zone guinéenne et zone soudanienne), une analyse de variance (Anova) a été effectuée en recourant au modèle linéaire généralisé pour les caractéristiques physiques et mécaniques. Le plan d'échantillonnage adopté correspond à un modèle mixte d'analyse de la variance, partiellement hiérarchisé. Le facteur aléatoire « arbre » est subordonné au facteur fixe « site ».

Ainsi, pour chaque paramètre, l'Anova a permis de spécifier la différence entre les moyennes des valeurs mesurées selon la provenance de l'arbre, les étoiles indiquant le niveau de significativité du test $\left({ }^{*} \mathrm{P}<0,05 ; * * \mathrm{P}<0,01\right.$; $\star \star \star P<0,001)$. La corrélation entre la densité et les propriétés mécaniques, et entre l'âge cambial et les propriétés physico-mécaniques, a été étudiée. L'influence du PSF sur les autres propriétés a été également mise en évidence.

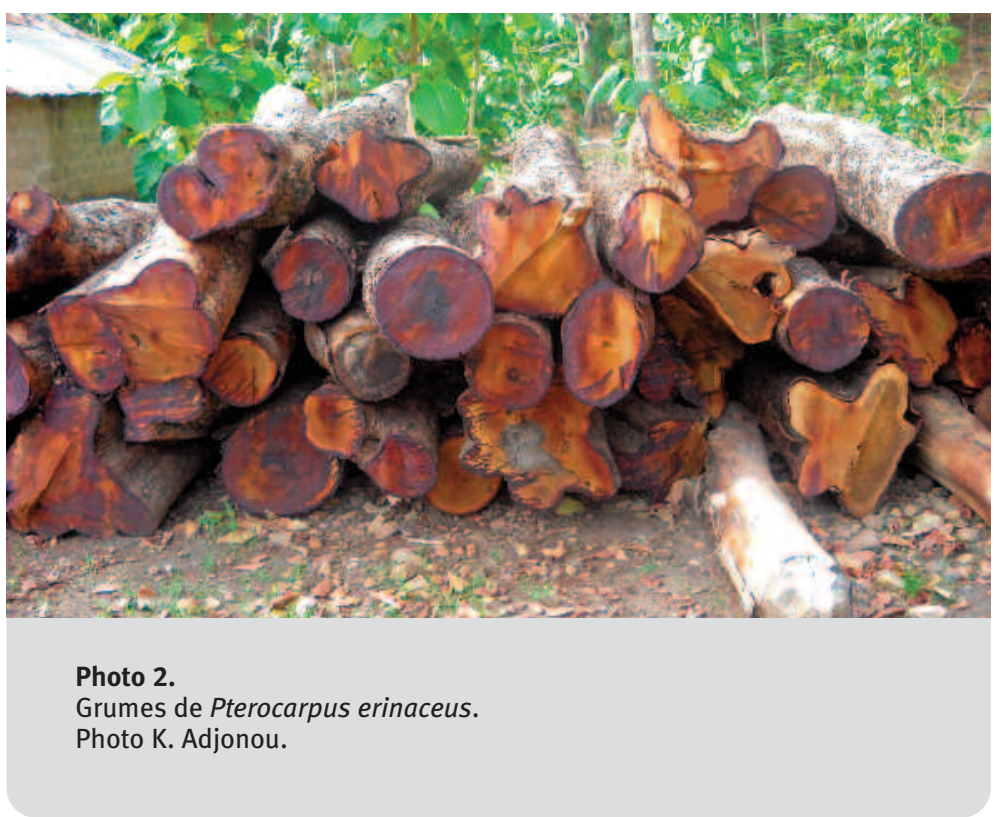




\section{Résultats}

\section{Variabilité des paramètres dendrométriques des arbres étudiés}

Le diamètre des arbres étudiés variait de 20,7 à $58,9 \mathrm{~cm}$ tandis que la hauteur du fût s'échelonnait de 1,7 à $9,5 \mathrm{~m}$. Ces arbres étaient âgés de 18 à 60 ans (tableau I). On note une nette différence de DBH (diamètre à 1,30 m du sol) et de hauteur du fût entre les deux sites de prélèvement. Le DBH et la hauteur du fût étaient en moyenne plus élevés à Abdoulaye qu'à Kéran (figure 3). Cette différence pourrait être expliquée par l'âge des arbres. En effet, les résultats indiquent qu'il y a une corrélation significative entre le DBH et l'âge des arbres $\left(P<0,05 ; R^{2}=0,6\right)$. En revanche, l'accroissement annuel de diamètre n'a pas montré de différence particulière entre les deux sites. L'Anova effectuée s'est révélée non significative ; toutefois, ces résultats ne sont le reflet que de quelques arbres.

Les densités d'arbres à l'hectare sont très proches entre les deux sites. On a dénombré $136 \pm 1,6$ tiges/ha dans la forêt d'Abdoulaye en zone guinéenne (Adjonou et al., 2010) et, lors de nos travaux réalisés sur quatre parcelles de $1000 \mathrm{~m}^{2}$, nous avons relevé $130 \pm 5,2$ tiges/ha dans le parc de la Kéran en zone soudanienne.

\section{Analyse des propriétés physiques et mécaniques du bois de vène tous arbres confondus}

Les retraits radial et tangentiel variaient respectivement de 1,63 à 5,37 \% et de 3,28 à $8,76 \%$ avec des moyennes respectives de $3,28 \% \pm 0,7$ et $5,76 \% \pm 1,0$. Le retrait surfacique s'élevait en moyenne à 9,2 $\pm 1,7 \%$. Le PSF, l'infra-densité et la densité ont en moyenne été estimés respectivement à 19,2 $\pm 2,7 \%, 0,76 \pm 0,06 \mathrm{~g} / \mathrm{cm}^{3}$ et $0,88 \pm 0,07 \mathrm{~g} / \mathrm{cm}^{3}$ (tableau II). La densité et l'infra-densité variaient peu, les coefficients de variation obtenus étant respectivement de $7,9 \%$ et $8,1 \%$. Les retraits radial et tangentiel et le PSF présentaient un coefficient de variation plus élevé, respectivement de $21,6 \%, 17,8 \%$ et $14,1 \%$. Le MOE, la dureté, la flexion et la compression présentaient un coefficient de variation respectif de $14,4 \%, 28,7 \%, 18,9 \%$ et $14,6 \%$.

\section{Variabilité des caractéristiques physiques}

\section{Densité à $12 \%$ et infra-densité}

En approche univariée, les diagrammes « boîte à moustache » des variables masse volumique et infra-densité semblaient montrer des différences en fonction des sites. La densité à $12 \%$ et l'infra-densité étaient respectivement de $0,88 \pm 0,08 \mathrm{~g} / \mathrm{m}^{3}$ et $0,75 \pm 0,01 \mathrm{~kg} / \mathrm{m}^{3}$ pour les arbres provenant de la forêt d'Abdoulaye. Pour les arbres provenant du parc OtiKéran, la densité à $12 \%$ et l'infra-densité étaient respectivement de $0,90 \pm 0,02 \mathrm{~kg} / \mathrm{m}^{3}$ et $0,78 \pm 0,01 \mathrm{~g} / \mathrm{m}^{3}$. Les mesures présentaient une variabilité plus élevée pour les arbres provenant du parc (figure 4). Pour ces deux paramètres, les valeurs étaient respectivement plus élevées de $4 \%$ et de $3 \%$ en zone soudanienne qu'en zone guinéenne. Ces résultats ont été confirmés par une analyse de variance significative $(P<0,01$ pour la densité et $P<0,03$ pour l'infra-densité).

\section{Retrait radial, retrait tangentiel, retrait surfacique, anisotropie, point de saturation des fibres, coefficient de retrait surfacique}

Les retraits radial et tangentiel ne montrent pas de différences particulières entre les deux sites $(P>0,05)$. Le retrait tangentiel était de 5,81 $\pm 0,96$ à Abdoulaye et de $5,64 \pm 1,06$ à Kéran. Le retrait radial était de 3,10 $\pm 0,78$ à Kéran et de $3,37 \pm 0,63$ à Abdoulaye. Cette absence de différence des retraits 

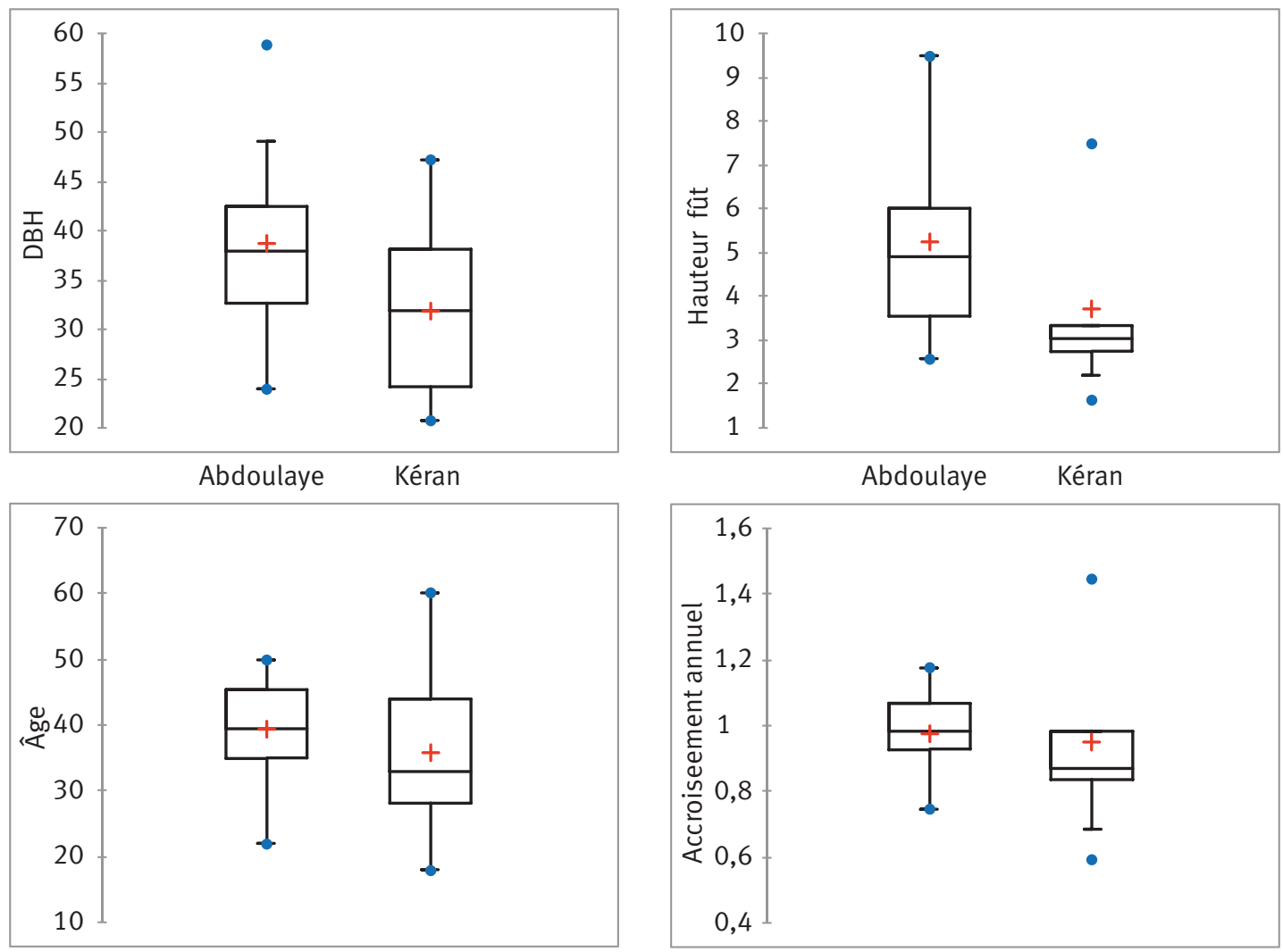

Abdoulaye

Kéran

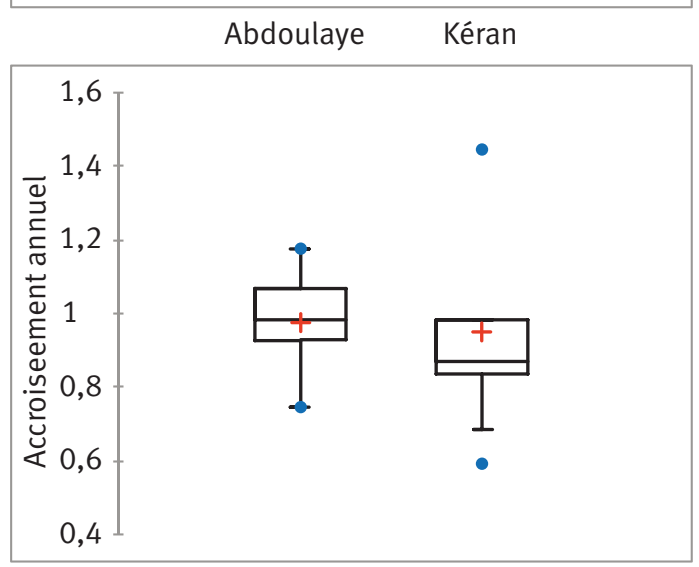

Abdoulaye Kéran

Figure 3.

Profils statistiques des caractéristiques dendrométriques en fonction des sites.

Tableau II.

Principales caractéristiques physiques et mécaniques du bois de Pterocarpus erinaceus.

\section{Propriétés}

Masse volumique $\left(\mathrm{g} / \mathrm{cm}^{3}\right)$

Infra-densité $\left(\mathrm{g} / \mathrm{cm}^{3}\right)$

Retrait T (\%)

Retrait R (\%)

Anisotropie

PSF (\%)

Retrait surfacique (\%)

Coefficient retrait surfacique

MOE spécifique (MPa)

MOE (MPa)

MOR (MPa)

Compression (MPa)

Dureté Monnin

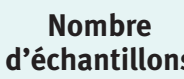

Moyenne

Écarttype

Minimum

Maximum

CV (\%)

d'échantillons

89

94

94

$94 \quad 3,2$

0,88

0,07

0,72

0,06

0,61

$1,0 \quad 3,2$

1,6

0,2

1,2

19,2

2,7

15,3

6,6

0,48

1,7

0,32

0,06

10892

1922

9717

26,5

85,8

10

3,7

49

5,8

1,12

0,92

8,7

5,3

2,2

25,0

13,9

0,61

22360

19127

206,5

18,9

89

70
12

5,8 


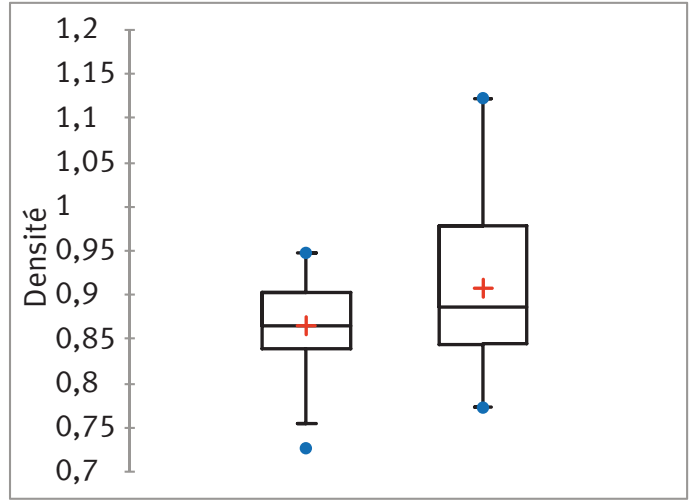

Abdoulaye

Kéran

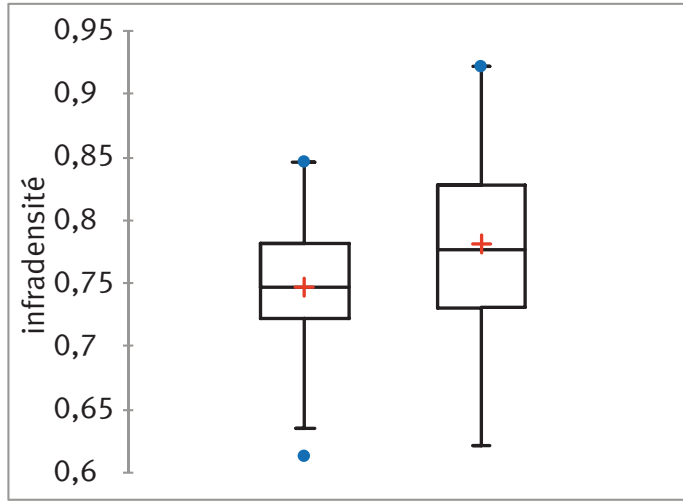

Abdoulaye Kéran

Figure 4.

Profils statistiques de la densité $\left(\mathrm{g} / \mathrm{cm}^{3}\right)$ et de l'infra-densité

$\left(\mathrm{g} / \mathrm{cm}^{3}\right)$ selon les deux sites.

radial et tangentiel entre les deux sites entraîne celle des retraits surfaciques entre les deux sites $(P>0,05)$. L'anisotropie de retrait montrait par contre une différence entre les deux sites ( $P=0,01)$, avec une valeur de $1,74 \pm 0,19$ à Abdoulaye et de 1,8 $\pm 0,24$ à Kéran (figure 5). Le PSF ne variait pas significativement d'un site à l'autre $(P>0,05)$. Il s'élevait à $19,06 \pm 2,93 \%$ à Kéran et $19,07 \pm 2,09 \%$ à Abdoulaye.

\section{Les caractéristiques mécaniques}

Le module d'élasticité (MOE) ne différait pas entre les deux sites (figure 6). Les moyennes étaient de $14150 \pm 1900 \mathrm{MPa}$ pour Abdoulaye et $14100 \pm 2300 \mathrm{MPa}$ pour Kéran. Cependant, les arbres provenant de la forêt d'Abdoulaye présentaient une variabilité moins étendue (cv $=13 \%)$ par rapport à ceux du parc de Kéran (cv=16\%). La même observation a été faite pour le module d'élasticité spécifique, qui ne variait pas significativement d'un site à l'autre $(P>0,05)$. La dureté variait de 5,8 à 23,8 avec une valeur moyenne de 12. La figure 6 montre qu'il n'y avait pas de différence particulière entre les deux sites $(P>0,3)$. Elle était en moyenne de 13,09 \pm 3,87 à Kéran et de 12,08 \pm 3,33 à Abdoulaye. Les valeurs des contraintes de rupture en compression et en flexion variaient respectivement de 49 à $95 \mathrm{MPa}$ et de 85,8 à 206,5 MPa. Seules les valeurs de la contrainte de rupture en compression variaient significativement en fonction des sites. Cette variable montrait une différence hautement significative $(P<0,001)$. Elle était de $66,5 \pm 9,4 \mathrm{MPa}$ à Abdoulaye et de 73,5 \pm 10,3 MPa à Kéran. La contrainte de rupture en flexion variait de $85,5 \mathrm{MPa}$ à 206,5 MPa. Elle ne révélait pas de différence particulière entre les deux sites (figure 6), ce résultat étant confirmé par une analyse de variance non significative $(P>0,2)$. Cette variable s'élevait en moyenne à $137,5 \pm 25,5 \mathrm{MPa}$ à Abdoulaye, et à $145 \pm 27,5 \mathrm{MPa}$ à Kéran.

\section{Relation entre l'âge et les propriétés technologiques du bois de vène (effet de la position radiale)}

L'analyse des propriétés mécaniques en fonction du nombre de cernes compté depuis la moelle a montré que seules les contraintes de rupture en compression et en flexion statique étaient corrélées avec l'âge cambial (figure 7). La zone la plus jeune ou de bois juvénile apparaissait légèrement plus "résistante » en compression et flexion que la zone du tronc plus âgée. Mais cette corrélation restait faible, comme le montrent les coefficients de détermination obtenus (compression : $R^{2}=0,058$; flexion : $R^{2}=0,093$ ). L'âge influencerait donc très peu les propriétés mécaniques du bois de vène.

Pour les propriétés physiques, seul le PSF était lié négativement à l'âge cambial (figure 8). La présence d'un taux d'extrait plus fort dans la zone centrale du tronc pourrait expliquer ce résultat ; cela concorde avec les résultats de Brémaud (2006) qui a montré l'influence du taux d'extrait notamment à l'alcool benzène sur le PSF (Brémaud et al., 2010). On note une très faible diminution de la densité en fonction de l'âge $\left(R^{2}=0,11\right)$, ce qui est intéressant au plan technologique. Enfin, d'une manière générale, comme pour les propriétés mécaniques, les propriétés physiques du bois de $P$. erinaceus restent très peu, voire non influencées par l'âge de l'arbre.

\section{Relation entre la densité et les propriétés mécaniques}

La figure 9 présente les résultats qui mettent en évidence les corrélations entre les propriétés mécaniques et la densité. L'analyse de cette figure montre qu'il n'y a pas de corrélation significative entre la densité et la contrainte de rupture en compression, d'une part, et entre la densité et le module d'élasticité, d'autre part. La densité était corrélée positivement à la contrainte à la rupture en flexion, avec un 

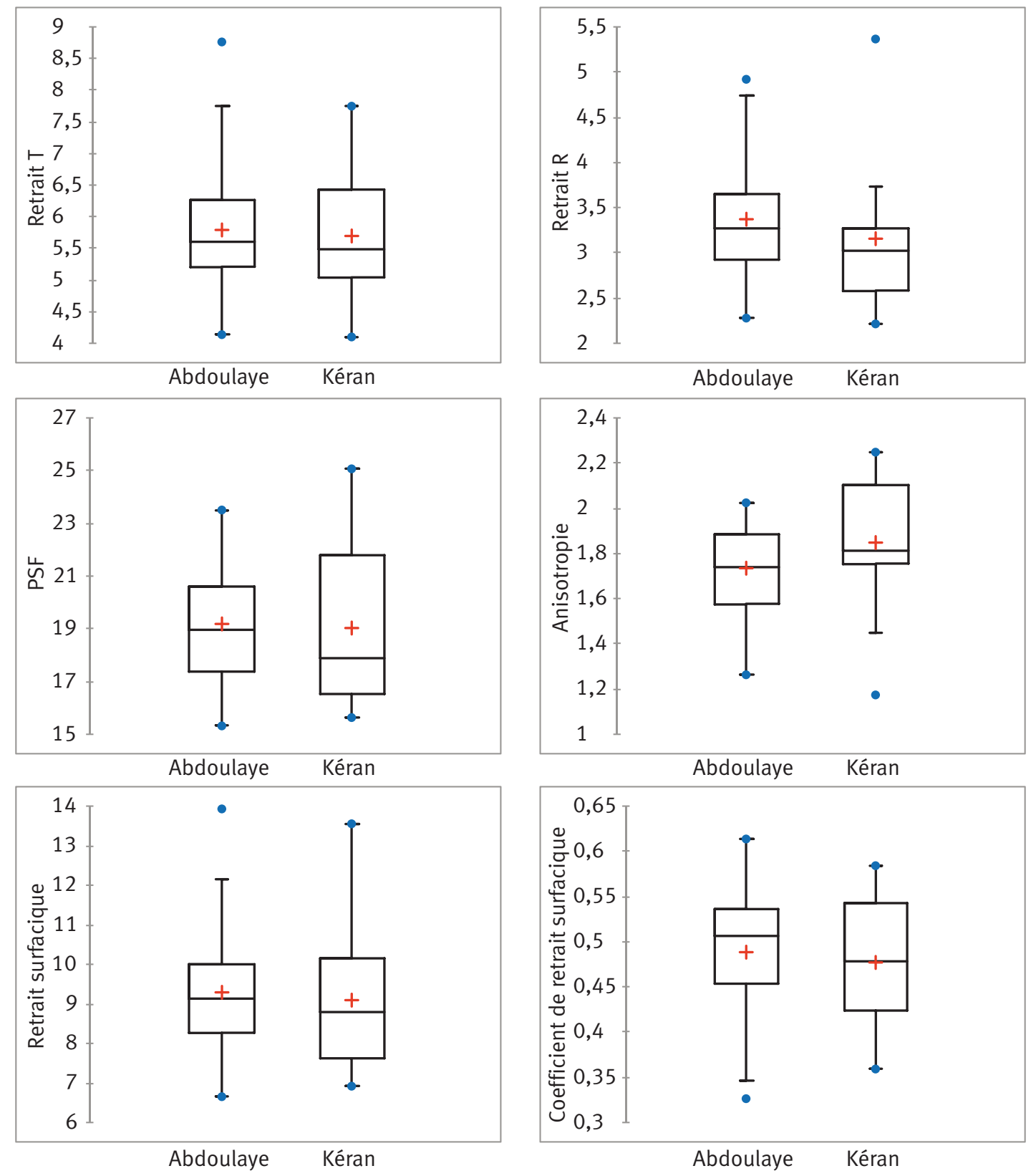

Figure 5.

Profils statistiques des retraits $T$ et $R$ (\%), de l'anisotropie des retraits, du retrait surfacique (\%), du PSF (\%) et du coefficient de retrait surfacique sur les deux sites.

\section{Relation entre le PSF et les autres propriétés}

coefficient de détermination de 0,43 $(P=0,001)$. La densité et la dureté Monnin étaient positivement et significativement corrélées avec un coefficient de détermination de $0,79(P=0,0001)$.
L'analyse des relations entre le PSF et les autres propriétés du bois de $P$. erinaceus indique une corrélation négative entre ce dernier et la densité et/ou l'infra-densité (tableau III). Ces résultats concordent avec ceux de Brémaud (2006) qui a travaillé sur différentes espèces tropicales utilisées pour la réalisation de xylophones (Brémaud et al., 2010). Par ailleurs, le PSF influe positivement sur les retraits tangentiel, radial et surfacique avec un effet plus marqué suivant la direction tangentielle (tableau III). 

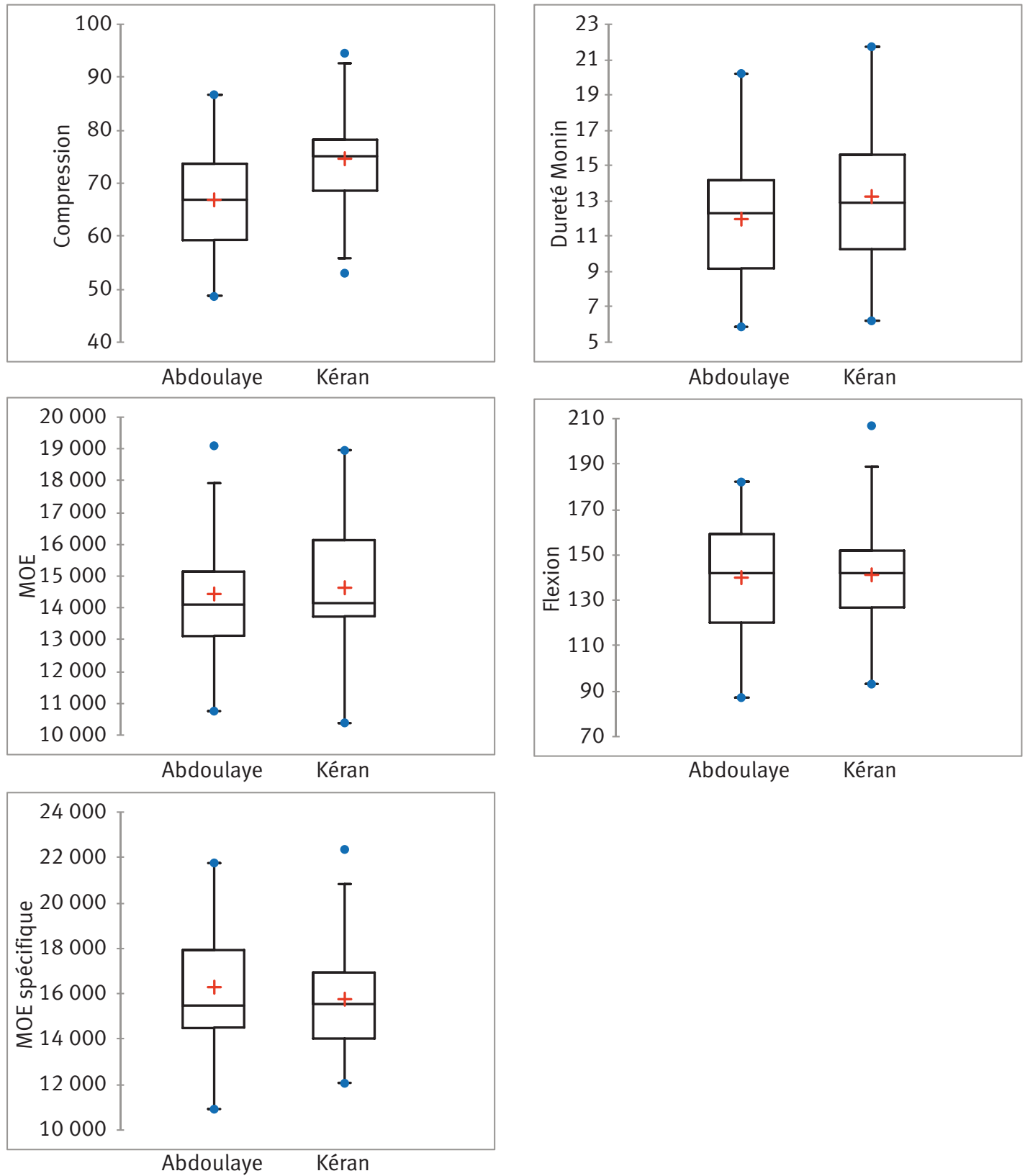

Abdoulaye

Kéran

Figure 6.

Profils statistiques permettant de comparer le MOE (MPa), le module d'élasticité spécifique, les modules de compression ( $\mathrm{MPa}$ ), de flexion (MPa) et la dureté Monnin.

\section{Discussion}

\section{Qualité du bois de vène}

Le bois de vène peut être classé comme bois lourd et très dur avec une densité de l'ordre de $0,88 \mathrm{~g} / \mathrm{cm}^{3}$. Avec des retraits tangentiel et radial faibles, respectivement de 5,8\% et $3,3 \%$, le bois est considéré comme stable avec une anisotropie de retrait inférieure à 2. La densité élevée du bois entraîne un séchage lent qui n'occasionne pratiquement pas de défauts grâce à ses faibles retraits. Il présente des contraintes moyennes de rupture en compression et en flexion statique respectivement de $70 \mathrm{MPa}$ et $140 \mathrm{MPa}$ et un module d'élasticité de l'ordre de 14500 MPa. Il affiche aussi un module d'élasticité spécifique élevé (16 070 MPa). Par ces propriétés, le bois de vène du Togo est tout à fait comparable à celui provenant du Sénégal et du Mali (base de données Cirad, non publiée). Selon Bork et Meyer (1985) 

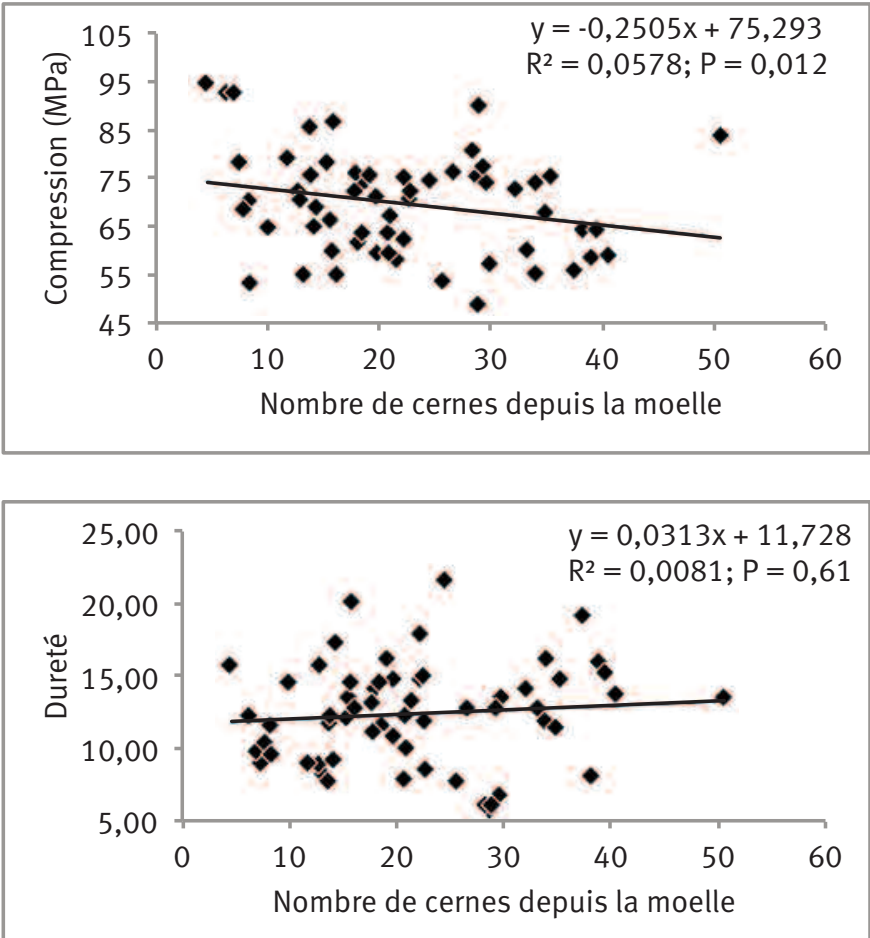
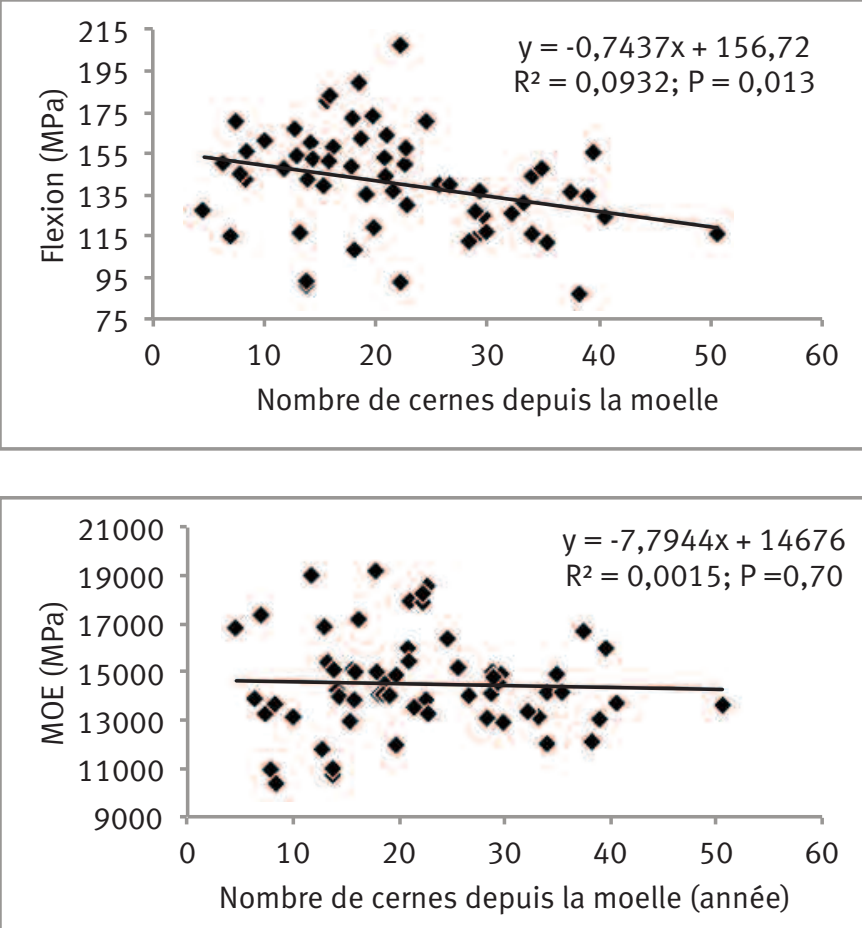

Figure 7.

Effet de la position radiale sur les propriétés mécaniques.

et Holz (1996), les espèces destinées à la production de xylophones sont de densité élevée, environ 0,80 à $0,95 \mathrm{~g} / \mathrm{cm}^{3}$, avec un module d'élasticité élevé, de l'ordre de 14 à $20 \mathrm{GPa}$. Par ailleurs, Ono et Norimoto (1983), Yano et al. (1994) et Brémaud (2006) ont montré que ces bois étaient moins sensibles aux variations d'humidité, tout en

Tableau III.

Corrélation entre le PSF et les autres propriétés.

\begin{tabular}{lccc|} 
Variables & $\begin{array}{c}\text { Coefficient de } \\
\text { corrélation Pearson }\end{array}$ & $\mathbf{R}^{\mathbf{2}}$ & $\boldsymbol{P}$ value \\
\hline Densité & $-0,584$ & 0,341 & $<0,0001$ \\
\hline Infra-densité & $-0,758$ & 0,574 & $<0,0001$ \\
\hline Dureté Monnin & $-0,6$ & 0,421 & $<0,0001$ \\
\hline Retrait T & 0,674 & 0,455 & $<0,0001$ \\
\hline Retrait R & 0,461 & 0,213 & 0,000 \\
\hline Anisotropie & 0,216 & 0,047 & 0,090 \\
\hline Retrait surfacique & 0,44 & 0,2 & 0,000 \\
\hline MOE & $-0,17$ & 0,029 & 0,183 \\
\hline Flexion & $-0,185$ & 0,034 & 0,147 \\
\hline Compression & 0,06 & 0,004 & 0,608 \\
\hline
\end{tabular}

présentant de faibles valeurs du coefficient d'amortissement associées à un module spécifique élevé. Pour ses propriétés connues empiriquement, le bois de vène est réputé et recherché, notamment pour la confection de xylophones en Afrique de l'Ouest et sahélienne.

\section{Variabilité des propriétés physiques et mécaniques}

Les résultats montrent que la densité et l'infra-densité diffèrent significativement entre les provenances. Ils concordent avec ceux obtenus sur le bois de vène provenant du Mali par Traoré qui a également révélé un effet site sur l'infra-densité du vène (Traoré, 2009). Dans son étude, il a trouvé en zones guinéenne et soudanienne des valeurs significativement différentes d'infra-densité s'élevant respectivement à $0,80 \mathrm{~g} / \mathrm{cm}^{3}$ et $0,75 \mathrm{~g} / \mathrm{cm}^{3}$. Il attribue cette variation entre stations à des modifications anatomiques et de la teneur en composés extractibles résultant de la différence de conditions de croissance des arbres, de leur patrimoine génétique, et de la qualité des sols. 

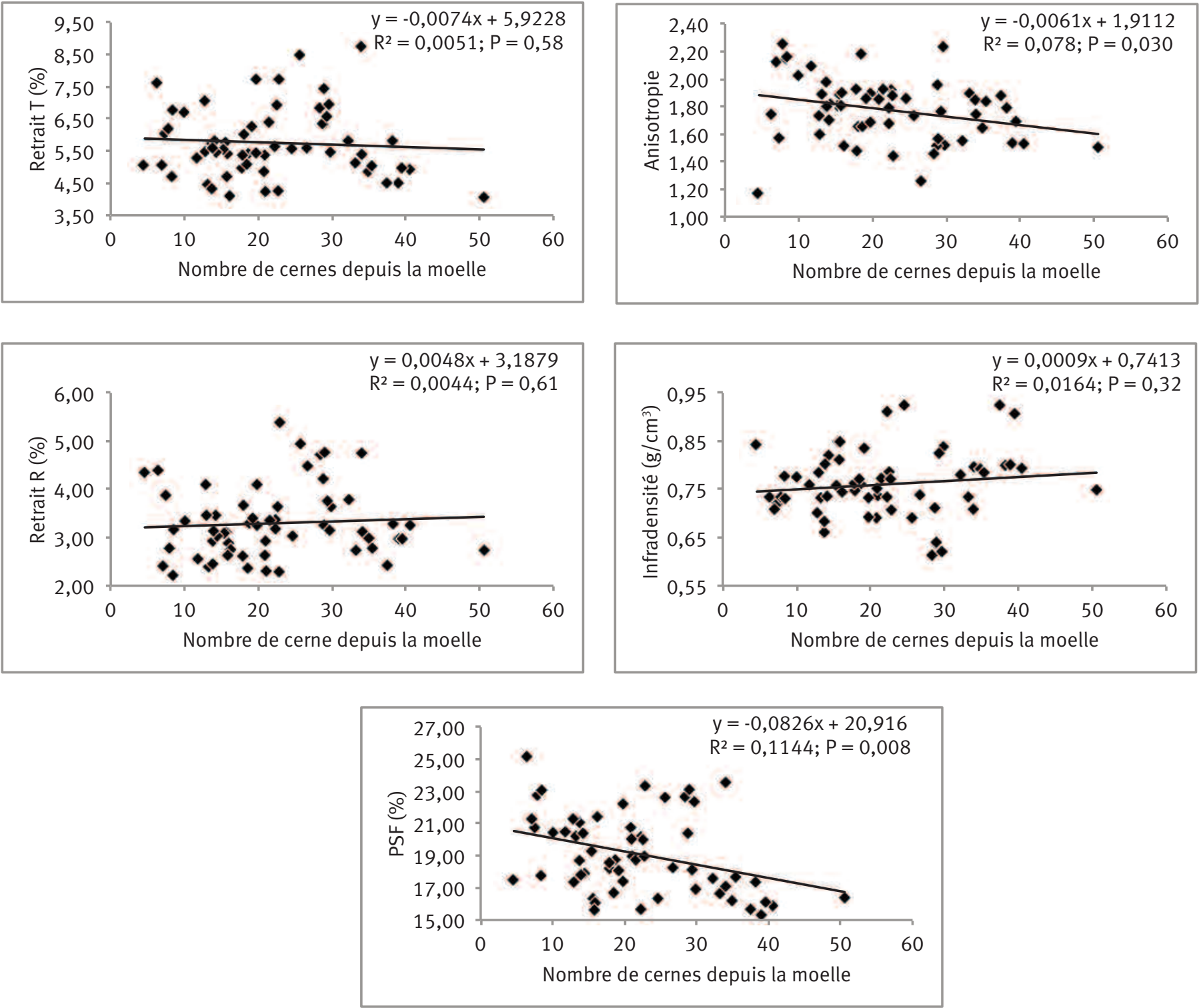

Figure 8.

Effet de la position radiale sur les propriétés physiques étudiées.

Certaines recherches effectuées sur des feuillus tropicaux ont montré des différences significatives de la masse volumique du bois entre les provenances et/ou descendances chez quelques espèces évaluées entre l'âge de 6 et 10 ans. Il s'agit, par exemple, des travaux réalisés sur Sterculia apetala (Jacq.) Karst. (Dvorak et al., 1998). Selon ces auteurs, tous les facteurs écologiques qui diminuent la fertilité des stations diminuent également la densité. Zobel et Jett (1995) ont montré qu'en plus des effets de l'environnement la masse volumique du bois pouvait varier significativement en fonction de la provenance et/ou de la famille botanique. S'agissant des autres propriétés physiques, les travaux de Traoré (2009) confirment une partie de nos résultats selon lesquels il n'y a pas de différence significative entre les retraits selon l'origine géographique. Cet auteur attribue cette constance des valeurs du retrait à la forte teneur en extraits du bois de vène, qui pourrait limiter l'effet direct des modifications anatomiques liées aux conditions de croissance et au vieillissement du cambium. Les extraits affectent ainsi le comportement hygroscopique général du bois et les espèces à haute teneur en extractibles se caractérisent par de faibles coefficients de rétractibilité et de faibles valeurs du point de saturation des fibres (Skaar, 1988 ; Hernandez et Restrepo, 1995). Allant dans le même sens, Traoré (2009) a montré qu'après élimination des extraits les retraits radial et tangentiel ainsi que le retrait 

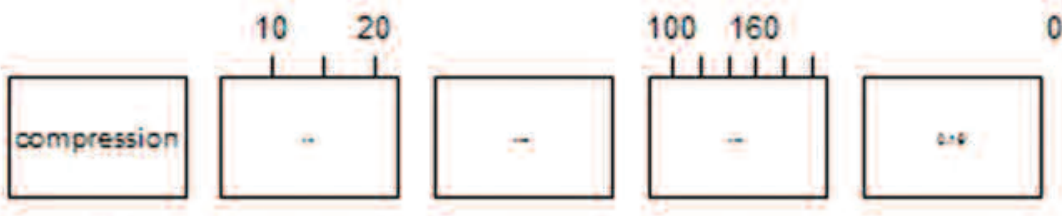

$0.60 \quad 0.80$
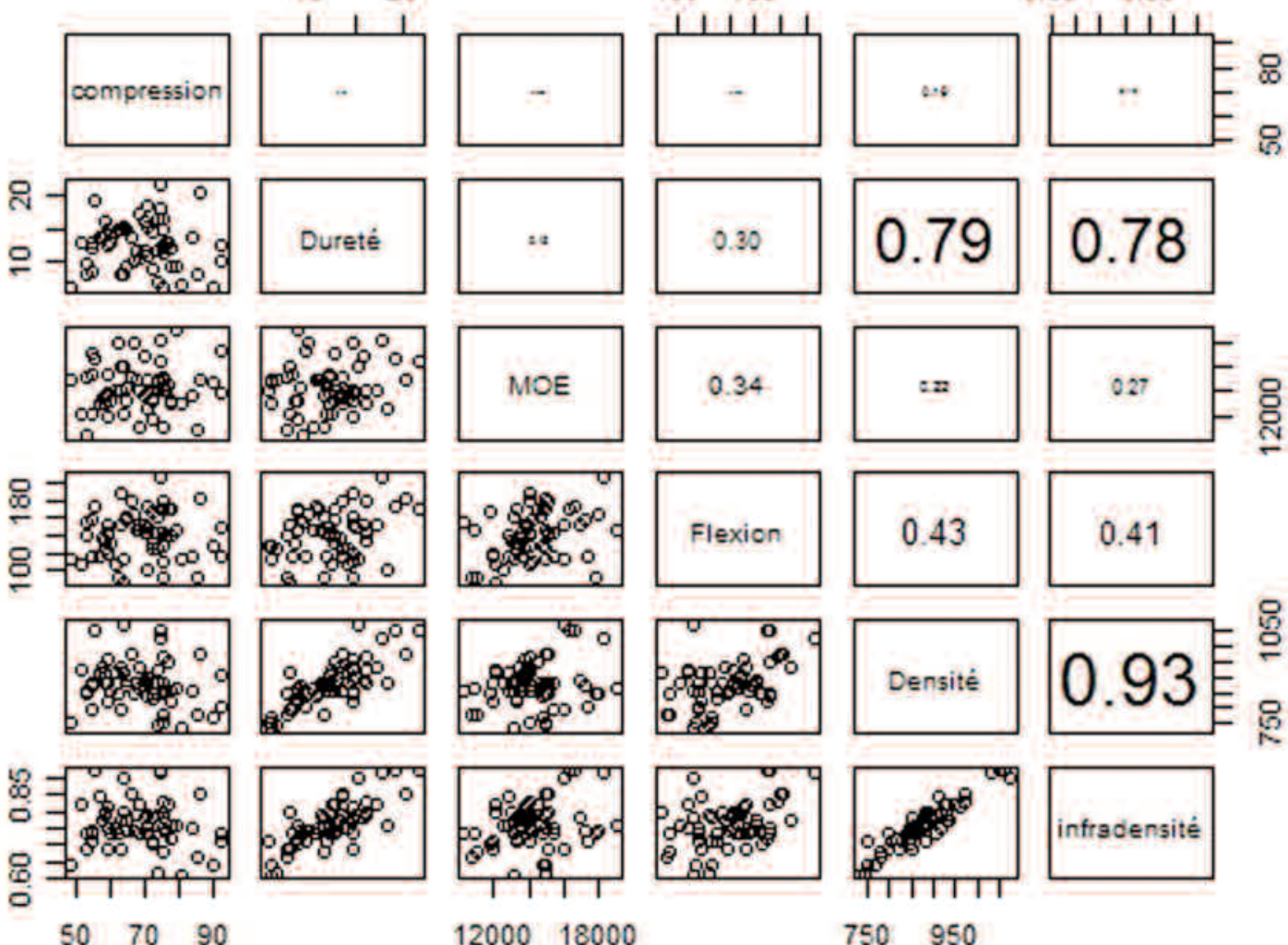

$12000 \quad 18000$

$750 \quad 950$

Figure 9.

Corrélations entre la densité et les propriétés mécaniques du bois de vène.

volumique augmentent de manière significative chez $P$. erinaceus. Des travaux similaires effectués par Choong et Achmadi (1991) ont montré que l'élimination des extraits à l'eau et aux solvants organiques entraînait une augmentation excessive du retrait.

L'anisotropie des retraits, rapport entre le retrait tangentiel et le retrait radial, est l'un des paramètres importants pour évaluer la stabilité dimensionnelle du bois et sa nervosité : plus sa valeur tend vers l'unité, moins le bois à tendance à se déformer en séchant (Traoré, 2009). Elle découle des modalités de croissance des arbres qui définissent les trois directions privilégiées d'étude d'un bois (Chanson, 1985). Les résultats montrent qu'elle varie entre les deux sites, donc en fonction des conditions de croissance. Selon Masserann et Mariaux (1985), le diamètre radial des fibres est considéré comme le facteur dominant de la variation de l'anisotropie des retraits. Dans la présente étude, les valeurs sont plus élevées à Kéran qu'à Abdoulaye. Il s'agirait de confirmer ces résultats par une analyse anatomique des propriétés des fibres de nos échantillons.

Les résultats ont montré que le retrait radial et le retrait tangentiel étaient liés et que l'anisotropie était significativement corrélée avec le retrait radial. Ces résultats sont identiques à ceux obtenus par Masserann et Mariaux (1985) qui ont trouvé chez Terminalia superba une corrélation forte et positive entre les retraits, et une corrélation forte et négative entre le retrait radial et l'anisotropie.
Parmi les propriétés mécaniques étudiées, seule la contrainte de rupture en compression varie significativement en fonction des sites. Le module d'élasticité est significativement supérieur chez les spécimens provenant de la zone guinéenne par rapport à ceux de la zone soudanienne. Comme il est signalé, la contrainte de rupture en compression et le module d'élasticité ne sont pas liés à la densité du bois dans cette étude. Ces résultats concordent avec ceux obtenus par Meite et al. (2010). Ces auteurs ont montré sur des bois de pin maritime et de pin d'Alep qu'il n'y avait pas de corrélations significatives entre les modules d'élasticité et la densité, et entre les contraintes de rupture en compression et la densité. Par contre, la dureté est significativement liée à la densité du bois. Cela montre que l'augmentation de la densité entraîne celle de la dureté.

Les résultats ont indiqué une corrélation significative négative entre le PSF et la densité et la dureté. En revanche, la corrélation est significative et positive entre le PSF et les retraits radial et tangentiel. En ce qui concerne l'effet de la position radiale, les résultats indiquent de façon générale qu'il n'y a pas de corrélation très significative entre l'âge cambial et les propriétés mesurées. Même s'il semble exister un lien entre la position radiale et le PSF et les contraintes de rupture en compression et en flexion, le PSF est faible et se traduit par les faibles coefficients de détermination obtenus (compression : $\mathrm{R}^{2}=0,057$; flexion : $\mathrm{R}^{2}=0,093$; PSF : $\left.\mathrm{R}^{2}=0,11\right)$. 


\section{Conclusion}

En conclusion, les résultats sur les caractéristiques physiques et mécaniques du bois de Pterocarpus erinaceus (Poir.) du Togo montrent que le bois présente des caractéristiques performantes pour des utilisations variées telles que la construction, la menuiserie, l'ébénisterie ou la fabrication d'instrument de musique. L'étude de la corrélation entre les propriétés mesurées fait apparaître que les caractéristiques mécaniques de cette espèce sont très peu influencées par la densité des bois. La densité ne serait donc pas un critère de sélection. L'étude a aussi mis en évidence une supériorité relative de certaines propriétés technologiques (densité, infra-densité, anisotropie des retraits et contrainte à la rupture en compression) des arbres provenant de Kéran (zone soudanienne). Enfin, ces résultats sont des informations utiles aux forestiers togolais quant au comportement de cette espèce susceptible de faire l'objet de programmes de plantation. Ils pourraient également permettre aux gestionnaires des ressources naturelles de mettre en place des parcelles de conservation dans des zones réservées au Togo.

\section{Remerciements}

Les auteurs remercient la Commission de l'Union économique et monétaire ouest-africaine (UEMOA) qui a financé cette recherche dans le cadre du projet Appui à l'enseignement supérieur dans les pays membres de l'UEMOA (PAES). Ils remercient aussi L'Agence Universitaire de la Francophonie (AUF) pour l'attribution d'une bourse de mobilité. Leurs remerciements vont également à l'Unité de recherche BioWooEB du Centre International de Recherche Agronomique pour le Développement (CIRAD) pour son appui matériel et financier et pour l'accueil de Monsieur Kossi Novinyo Segla. Les auteurs remercient Monsieur Pierre Détienne pour son appui technique.

\section{Références bibliographiques}

Adjonou K., Napo A., Kokutse A., Segla K., Kokou K., 2010. Étude de la dynamique des peuplements naturels de Pterocarpus erinaceus Poir. (Fabaceae) surexploités au Togo. Bois et Forêts des Tropiques, 306 : 45-56. [En ligne] http://bft.cirad.fr/cd/ BFT_306_45-55.pdf

AFNOR, 1988. Bois et liège : recueil de normes françaises. Paris, France, Association française de normalisation, 163-207.

Bordonne P. A., 1989. Module dynamique et frottement intérieur dans le bois : mesures sur poutres flottantes en vibrations naturelles. Thèse de doctorat en Sciences du bois, Institut national polytechnique de Lorraine, France, 109 p.

Bork I., Meyer J., 1985. On the tonal evaluation of xylophones. Braunschweig, Allemagne, Physikalisch-Technische Bundesanstalt.

Brémaud I., 2006. Diversité des bois utilisés ou utilisables en facture d'instruments de musique. Thèse, Université Montpellier II, France.

Brémaud I., Minato K., Langbour P., Thibaut B., 2010. Physicomecanical indicators of interspecific variability in vibration damping of wood. Annals of Forest Science, 67 (7): 107-114.
Chanson B., 1985. La variabilité du bois : exemple des taillis de châtaigniers méditerranéens Castanea sativa Mill. Forêt Méditerranéenne, 10 (1) : 293-296.

Choong E. T., Achmadi S., 1991. Effect of extractives on moisture sorption and shrinkage in tropical woods. Wood and Fiber Science, 23 (2): 185-196.

Dvorak W. S., Uruena H., Moreno L. A., Goforth H., 1998. Provenance and family variation in Sterculia apetala in Colombia. Forest Ecology and Management, 111: 127-135.

FAO, 2011. Situation des forêts du monde 2011. Rome, Italie, FAO, Étude FAO, 193 p. http://www.fao.org/docrep/013 /i2000f/i2000f.pdf.

Guitard D., 1987. Mécanique du matériau bois et composites. Toulouse, France, Cépaduès Éditions, 238 p.

Hernandez R. E., Restrepo G., 1995. Natural variation in wood properties of Alnus acuminata H.B.K. grown in Colombia. Wood and Fiber Science, 27 (1): 41-48.

Holz D., 1996. Acoustically important properties of xylophone-bar materials: can tropical woods be replaced by European species? Acta Acustica, 82: 878-884.

Kokou K., Nuto Y., Astri H., 2009. Impact of charcoal production on woody plant species in West Africa: A case study in Togo. Scientific Research and Essay, 4 (8): 881-893.

Masserann C., Mariaux A., 1985. Anisotropie de retrait et structure du bois : recherche de l'influence des caractères morphologiques transverses des fibres. Bois et Forêts des Tropiques, $209: 35-47$. [En ligne] http://bft.cirad.fr/cd/ BFT_209_35-47.pdf

Meite M., Laanaa A., Famiri A., Yeznasni A., Chergui M., El Ghorba M., 2010. Deuxième rapport d'évaluation de la gestion durable des forêts (GDF) du Togo (2005-2009).

MERF/OIBT, 2010. Deuxième rapport d'évaluation de la gestion durable des forêts (Gdf) du Togo (2005-2009), 56 p.

Ono T., Norimoto M., 1983. Anisotropy of dynamic young's modulus and internal friction in wood. Japan Journal of Applied Physics, 24 (8): 960-964.

Skaar C., 1988. Wood-water relations. New York, USA, Springer-Verlag, 283 p.

Timoshenko S., Goodier J. N., 1951. Theory of Elasticity. New York, USA, McGraw Hill, 506 p.

Traoré B., 2009. Influence de la structure anatomique et des métabolites secondaires sur les propriétés physiques et la qualité acoustique du bois de vène provenant du Mali. Thèse de doctorat, Université Laval, Québec, Canada, 206 p.

Traoré B., Brancheriau L., Perré P., Stevanovic T., Diouf P., 2010. Acoustic quality of vène wood (Pterocarpus erinaceus Poir.) for xylophone instrument manufacture in Mali. Annals of Forest Science, 67 (8): 815.

Yano H., Kajita H., Minato K., 1994. Chemical treatment of wood for musical instruments. Journal of the Acoustical Society of America, 96 (6): 3380-3391.

Zobel B. J., Jett J. B., 1995. Genetics of wood production. Berlin, Allemagne, Springer-Verlag, Springer Series in Wood Science, $337 \mathrm{p}$. 